\title{
Signo del vaso denso
}

\section{Dense Vessel Sign}

\author{
$\begin{array}{lll}\text { M. P. Seguí } & \text { E. N. Mayor } & \\ & \text { M. V. } \text { Clapsos }^{1}\end{array}$ \\ ${ }^{1}$ HIGA San Martín, La Plata, Argentina
}

Rev Argent Radiol 2018;82:190-192.

El signo del vaso denso en tomografía computada (TC) fue descripto por primera vez en el año 1981 por Yock, ${ }^{1}$ y consiste en una hiperdensidad espontánea y asimétrica de un vaso arterial, generalmente de la arteria cerebral media (ACM), debido a la oclusión de la misma por un trombo o un embolo. Ese hallazgo representa uno de los signos principales de accidente cerebro vascular (ACV) isquémico en el período hiperagudo. ${ }^{2} \mathrm{Si}$ bien el mismo es altamente específico, su sensibilidad es baja, encontrándose sólo en el $30 \%$ de los casos. $^{3}$

El ACV es una importante causa de muerte en la población adulta y la primera causa de invalidez en nuestro país. Se distinguen dos tipos: isquémico y hemorrágico. El primero constituye el $80 \%$ de los casos y dentro de las causas se mencionan la oclusión de un vaso arterial, ya sea por un trombo o un émbolo, lesiones tóxicas o anóxicas, hipotensión arterial mantenida, siendo la primera la principal etiología del ACV isquémico. ${ }^{3}$

La evolución de un $\mathrm{ACV}$ isquémico se divide en un periodo hiperagudo precoz, desde el inicio hasta las 6 horas (-Fig. 1); hiperagudo tardío, a partir de las 6 horas hasta las 24 horas; agudo, que abarca la primera semana (-Fig. 2); subagudo, dentro del primer mes; y crónico, después del primer mes de ocurrido el evento. ${ }^{4}$ Debido a la oclusión arterial total se desencadenan una serie de mecanismos que culminan en la falla de la bomba ATPasa sodio-potasio, con producción de edema citotóxico. Esos eventos se traducen en los estudios de imagen como signos precoces de isquemia. ${ }^{2}$

Ante la presencia de un déficit neurológico agudo el primer método indicado por ser el de mayor disponibilidad, de rápida de ejecución y eficacia es la TC de encéfalo sin contraste endovenoso. Permite descartar en primer lugar hemorragia o alguna otra patología que pueda simular isquemia, como tumores, colecciones, malformaciones vasculares, etc.; por otro lado detecta signos precoces o indirectos de isquemia. Dentro de esos últimos se mencionan la hipodensidad de ganglios basales y/
Address for correspondence M. P. Seguí, HIGA San Martín, La Plata, Argentina (e-mail: mpsegui_89@hotmail.com).

o del parénquima cerebral, el borramiento de los surcos de la convexidad, pérdida de diferenciación córtico-subcortical, cuyo mecanismo de producción es el edema citotóxico, y el signo del vaso denso o de la arteria cerebral media hiperdensa ( - Fig. 1). ${ }^{2}$

La presencia de una hiperdensidad en la arteria cerebral media de forma asimétrica, es decir, más hiperdensa que la ACM contralateral, representa uno de los signos más precoces y es causado por su oclusión trombótica o embólica. Ello se debe a que la densidad de la sangre en TC se relaciona con la concentración de hemoglobina. En ese sentido, la atenuación de la sangre circulante es de aproximadamente 40 Unidades Hounsfield (UH), mientras que la de un trombo intraluminal alcanza las $80 \mathrm{UH}$, motivo de su visualización hiperdensa. Si bien ese hallazgo se describe más comúnmente siguiendo el curso del segmento M1 de la arteria cerebral media, desde su origen hasta la cisura silviana, también puede evidenciarse en la arteria carótida interna y a nivel del tronco basilar. ${ }^{2}$

Dentro de los diagnósticos diferenciales del signo del vaso hiperdenso se distinguen calcificaciones vasculares, hiperdensidad secundaria a un hematocrito alto y dolicoectasia en el territorio de la circulación cerebral posterior. Asimismo, es importante descartar que no corresponda a un artefacto. ${ }^{2}$ Para prevenir un falso positivo se debe tener en cuenta que la ACM sea más densa que la contralateral y que cualquier otro vaso intracraneal de similar tamaño, así como que la alta densidad no sea debido a calcificación de la pared. ${ }^{2}$

Cabe destacar entre los diagnósticos diferenciales a los émbolos cerebrales calcificados. Esos son frecuentemente pequeños $(2-3 \mathrm{~mm})$ y pueden pasar fácilmente desapercibidos o ser confundidos con otras patologías, sobre todo en la TC inicial. Las causas más comunes de diagnósticos erróneos incluyen hemorragia, calcificación de la pared vascular o residuos infecciosos (neurocisticercosis). Todos los émbolos calcificados tienen received

September 7, 2017

accepted

January 27, 2018

published online

April 30, 2018
DOI https://doi.org/

$10.1055 / \mathrm{s}-0038-1641134$

ISSN 1852-9992.
Copyright (c) 2019, Sociedad Argentina de Radiología. Publicado por Thieme Revinter Publicações Ltda., Rio de Janeiro, Brazil. Todos los derechos reservados.
License terms

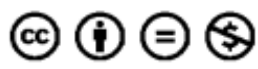



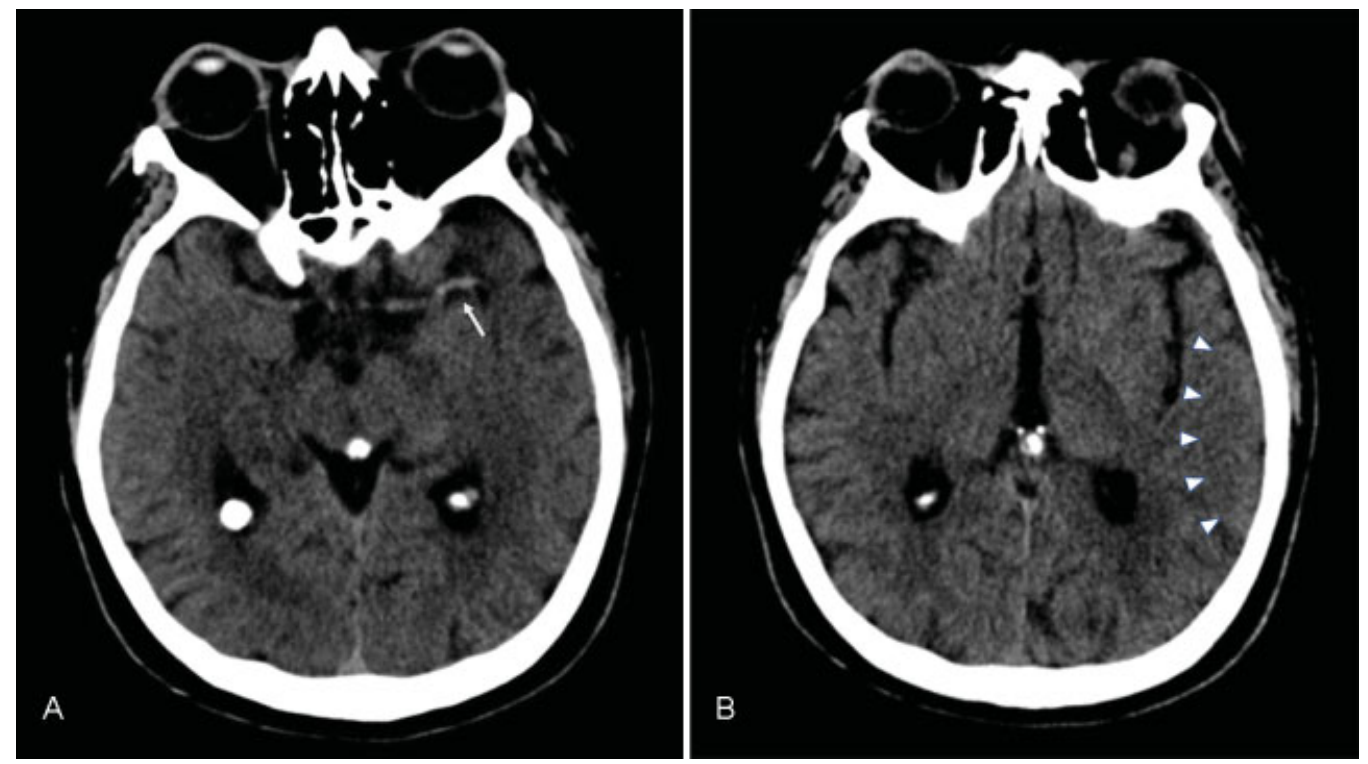

Fig. 1 Cortes axiales de tomografía de cerebro sin contraste endovenoso, de un paciente con déficit neurológico de pocas horas de evolución. Se observa una hiperdensidad espontánea en la arteria cerebral media izquierda (flecha blanca) a nivel temporal (A), con mínimo borramiento de los surcos regionales, señalado con cabezas de flecha blanca (B). Esos hallazgos son compatibles con signos hiperagudos de accidente cerebro vascular (ACV) isquémico.

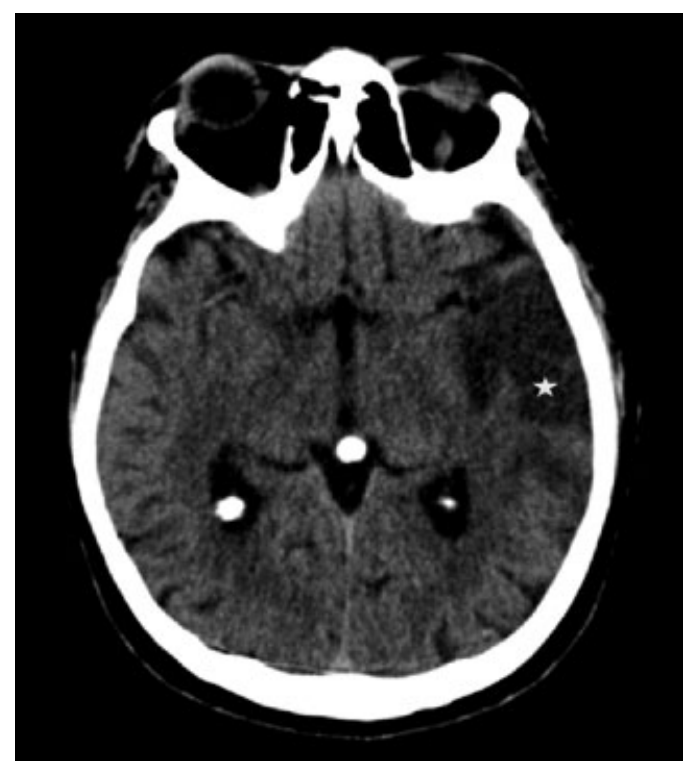

Fig. 2 El mismo paciente que en la figura 1, cuatro días después. En el corte axial de tomografía de cerebro sin contraste, se reconoce hipodensidad en territorio temporal izquierdo (estrella blanca), con aplanamiento de los surcos regionales, como expresión de ACV isquémico en evolución.

una atenuación significativamente mayor (162 UH) que los trombos intraluminales (50-70 $\mathrm{UH})$ y son redondeados $\mathrm{u}$ ovales en lugar de tubulares o lineales. ${ }^{5}$

\section{Conclusión}

El signo del vaso denso representa un hallazgo precoz altamente específico en el diagnóstico de ACV isquémico. Sin embargo, debido a su baja sensibilidad, se hace imprescindible tener en cuenta los otros signos concomitantes durante el período hiperagudo, para el diagnóstico, pronóstico y la instauración del tratamiento oportuno.

\section{Confidencialidad de los Datos}

Los autores declaran que han seguido los protocolos de su centro de trabajo sobre la publicación de datos de pacientes y que todos los pacientes incluidos en el estudio han recibido información suficiente y han dado su consentimiento informado por escrito.

\section{Responsabilidades Éticas}

Protección de personas y animales. Los autores declaran que para esta investigación no se han realizado experimentos en seres humanos ni en animales.

Confidencialidad de los datos. Los autores declaran que han seguido los protocolos de su centro de trabajo sobre la publicación de datos de pacientes.

Derecho a la privacidad y consentimiento informado. Los autores declaran que en este artículo no aparecen datos de pacientes.

\section{Declaración de Autoría}

Los autores somos responsables de la autoría del signo radiológico enviado.

\section{Conflicto de Intereses}

Los autores declaran no tener ningún conflicto de intereses.

\section{Agradecimientos}

A las Dras. Jimena Mariano y Estefanía Reyna. 


\section{Bibliografía}

1 Yock DH Jr. CT demonstration of cerebral emboli. J Comput Assist Tomogr 1981;5(02):190-196

2 Romero Vidal F. Isquemia e infarto cerebral. En: Mercader Sobrequés JM, Viñuela F. Neurorradiología diagnóstica y terapéutica. Barcelona: Masson; 2004;4:89-119

3 Srinivasan A, Goyal M, Al Azri F, Lum C. State-of-the-art imaging of acute stroke. Radiographics 2006;26(Suppl 1):S75-S95
4 Allen LM, Hasso AN, Handwerker J, Farid H, Sequence-specific MR. Sequence-specific MR imaging findings that are useful in dating ischemic stroke. Radiographics 2012;32(05):1285-1297, discussion 1297-1299

5 Walker BS, Shah LM, Osborn AG. Calcified cerebral emboli, a "do not miss" imaging diagnosis: 22 new cases and review of the literature. AJNR Am J Neuroradiol 2014;35(08):1515-1519 